\title{
COVID-19 vaccination in pregnancy
}

\author{
Antonio Vitiello $^{1} \mathbb{D} \cdot$ Raffaele La Porta $^{2} \cdot$ Valentina Troiano $^{2} \cdot$ Francesco Ferrara $^{1}$
}

Accepted: 2 May 2021 / Published online: 6 May 2021

(c) The Author(s), under exclusive licence to Springer Nature Switzerland AG 2021

\section{Dear Editor,}

The COVID-19 global pandemic, caused by the new SARS$\mathrm{CoV}-2$ Coronavirus, is still ongoing. A massive worldwide vaccination campaign began a few months ago [1]. Pharmacological therapeutic treatments are aimed at avoiding serious complications of the infection; antivirals directed against the SARS-CoV-2 virus are not yet available [2-4]. Certain population groups, such as the elderly and individuals with comorbidities, are at increased risk of severe infection. Evidence shows that pregnant women may also be at increased risk; this population category is particularly vulnerable to emerging infectious pathogens because of the alterations in immune and respiratory physiology that occur during pregnancy [5]. Therefore, preventing COVID-19 infection with vaccination can be important both for the mother and for the foetus. To date, vaccines with different mechanisms of action (e.g. mRNA, viral vector and protein subunit) are available, either authorised or investigational. Unfortunately, to date, clinical data supporting the efficacy and safety of COVID-19 vaccines in pregnant and lactating women are limited, due to the exclusion of pregnant women from pre-registration trials. For this reason, no clear recommendations about COVID-19 vaccination in pregnancy and lactation have been issued yet $[6,7]$. The Center for Disease Control and Prevention (CDC) and the FDA have activated systems to monitor the safety

Antonio Vitiello

antonio.vitiello2@uslumbria1.it

Raffaele La Porta

raffaele.laporta@sanita.marche.it

Valentina Troiano

valentinaelisaaa@gmail.com

Francesco Ferrara

francesco.ferrara@uslumbria1.it

1 Pharmaceutical Department, USL Umbria 1, Via XIV Settembre, Perugia, Italy

2 Pathology Department, ASUR Marche, Area Vasta 1, Urbino, Italy of COVID-19 vaccines during pregnancy and will closely monitor this information. Preliminary data from these systems are reassuring, and have not identified any safety concerns for pregnant people or their babies. In addition, the World Health Organization (WHO) suggests considering vaccinating pregnant women at high risk for infection (e.g. health care workers) or who have comorbidities. Recently, rare cases of thrombosis associated with thrombocytopenia have been identified following administration of viral vector COVID-19 vaccines [8]. Given that pregnant women have a higher rate of developing thrombosis than the general population [9], more clinical data in this patient population is warranted. There is a clear concern about receiving COVID19 vaccination in pregnancy and lactation given the lack of efficacy and safety data from available vaccines in this patient population. Therefore, it is critical to derive postmarketing data to monitor the safety profile of these vaccinations for pregnant and lactating women in actual clinical practice through the implementation of rigorously designed studies.

\section{Declarations}

Funding None.

Conflict of interest None of the authors have conflicts of interest to disclose.

Availability of data and materials Full availability of data and materials.

\section{References}

1. Vitiello A, Porta R, Pianesi L, et al. COVID-19 pandemic: vaccine and new monoclonal antibodies, point of view. Ir J Med Sci. 2021;12:1-2.

2. Ferrara F, Vitiello A. Scientific hypothesis for treatment of COVID-19's lung lesions by adjusting ACE/ACE2 imbalance. Cardiovasc Toxicol. 2021;21(6):498-503. 
3. Vitiello A, Pelliccia C, Ferrara F.Drugs acting on the renin-angiotensin system and SARS-CoV-2. Drug Discov Today. 2021; S1359-6446(21)00037-4.

4. Ferrara F, Vitiello A. Efficacy of synthetic glucocorticoids in COVID-19 endothelites. Naunyn Schmiedebergs Arch Pharmacol. 2021; $1-5$.

5. Dashraath P, Wong JLJ, Lim MXK, et al. Coronavirus disease 2019 (COVID-19) pandemic and pregnancy. Am J Obstet Gynecol. 2020;222(6):521-31.

6. Adhikari EH, Spong CY. COVID-19 vaccination in pregnant and lactating women. JAMA. 2021;325(11):1039-40.
7. Vora KS, Sundararajan A, Saiyed S, et al. Impact of COVID-19 on women and children and the need for a gendered approach in vaccine development. Hum Vaccin Immunother. 2020;16(12):2932-7.

8. Oldenburg J, Klamroth R, Langer F, et al. Diagnosis and management of vaccine-related thrombosis following AstraZeneca COVID-19 vaccination: guidance statement from the GTH. Hamostaseologie. 2021. https://doi.org/10.1055/a-1469-7481.

9. James AH. Thrombosis in pregnancy and maternal outcomes. Birth Defects Res C Embryo Today. 2015;105(3):159-66. 